Provided for non-commercial research and education use. Not for reproduction, distribution or commercial use.

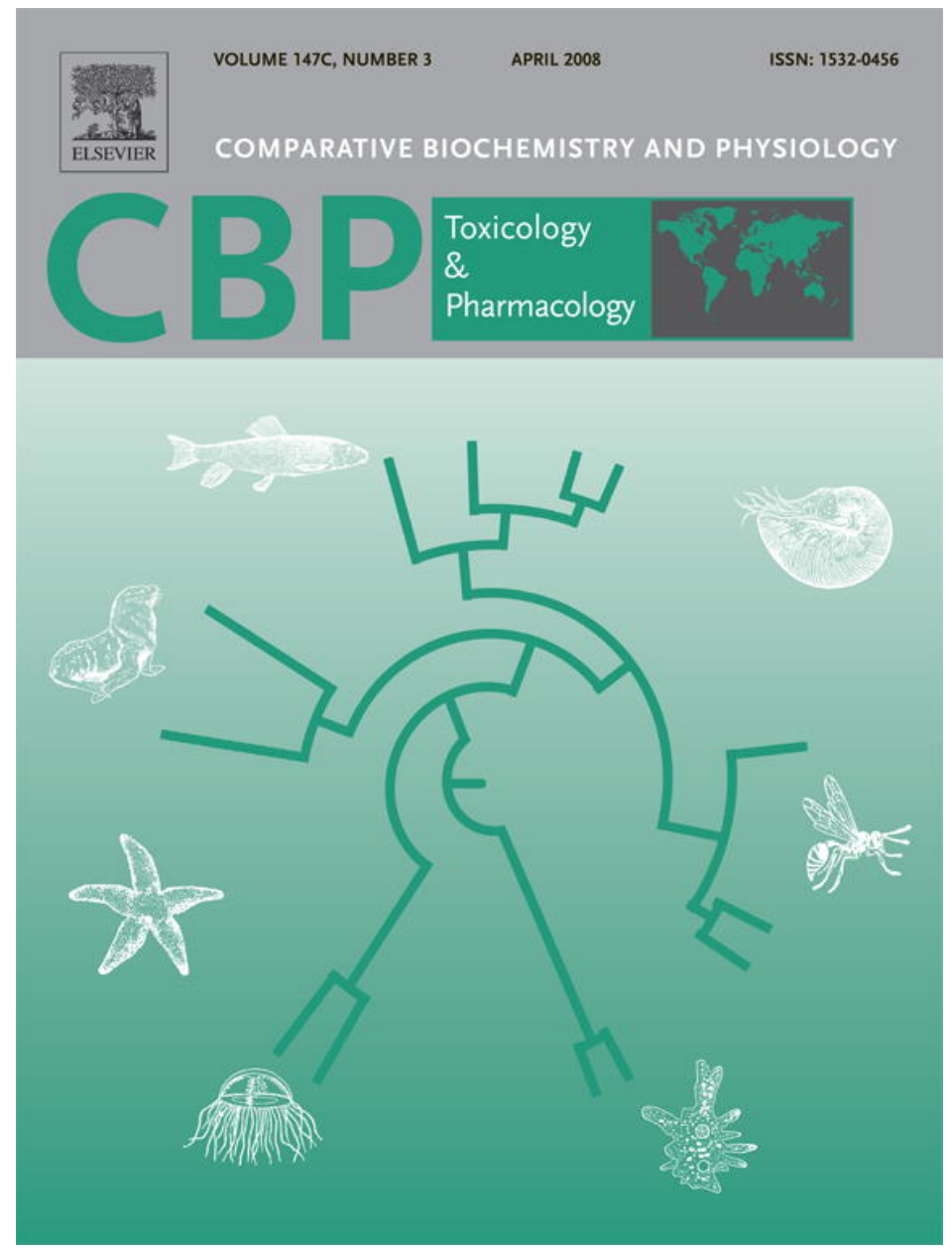

This article was published in an Elsevier journal. The attached copy

is furnished to the author for non-commercial research and education use, including for instruction at the author's institution, sharing with colleagues and providing to institution administration.

Other uses, including reproduction and distribution, or selling or licensing copies, or posting to personal, institutional or third party websites are prohibited.

In most cases authors are permitted to post their version of the article (e.g. in Word or Tex form) to their personal website or institutional repository. Authors requiring further information regarding Elsevier's archiving and manuscript policies are encouraged to visit:

http://www.elsevier.com/copyright 


\title{
Effects of phosphine on the neural regulation of gas exchange in Periplaneta americana
}

\author{
James D. Woodman ${ }^{\mathrm{a}, \mathrm{b}, *}$, Victoria S. Haritos ${ }^{\mathrm{b}}$, Paul D. Cooper ${ }^{\mathrm{a}}$ \\ a School of Botany and Zoology, Australian National University, Canberra ACT 0200, Australia \\ ${ }^{\mathrm{b}}$ CSIRO Entomology, GPO Box 1700, Canberra ACT 2601, Australia \\ Received 22 August 2007; received in revised form 31 October 2007; accepted 1 November 2007 \\ Available online 17 November 2007
}

\begin{abstract}
Phosphine is used for fumigating stored commodities, however an understanding of the physiological response to phosphine in insects is limited. Here we show how the central pattern generator for ventilation in the central nervous system (CNS) responds to phosphine and influences normal resting gas exchange. Using the American cockroach, Periplaneta americana, that perform discontinuous gas exchange (DGE) at rest, we simultaneously measure ventilatory nervous output from the intact $\mathrm{CNS}, V \mathrm{CO}_{2}$ and water loss from live specimens. Exposure to 800 ppm phosphine at $25{ }^{\circ} \mathrm{C}$ for $2 \mathrm{~h}(n=13)$ during recording did not cause any mortality or obvious sub-lethal effects. Within $60 \mathrm{~s}$ of introducing phosphine into the air flow, all animals showed a distinct CNS response accompanied by a burst release of $\mathrm{CO}_{2}$. The initial ventilatory response to phosphine displaced DGE and was typically followed by low, stable and continuous $\mathrm{CO}_{2}$ output. CNS output was highest and most orderly under normoxic conditions during DGE. Phosphine caused a series of ventilatory CNS spikes preceding almost complete cessation of CNS output. Minimal CNS output was maintained during the $2 \mathrm{~h}$ normoxic recovery period and DGE was not reinstated. $V \mathrm{CO}_{2}$ was slightly reduced and water loss significantly lower during the recovery period compared with those rates prior to phosphine exposure. A phosphine narcosis effect is rejected based on animals remaining alert at all times during exposure.
\end{abstract}

(c) 2007 Elsevier Inc. All rights reserved.

Keywords: CNS; Discontinuous gas exchange; Respirometry; Metathoracic ganglion

\section{Introduction}

Phosphine gas $\left(\mathrm{PH}_{3}\right)$ is the most widely used stored commodity fumigant as it is easy to apply, inexpensive, readily available, highly effective and leaves minimal residues in treated products (Chaudhry, 1997). The near complete reliance upon phosphine worldwide has been exacerbated in recent times by the international agreement to phase out use of methyl bromide (United Nations Environment Programme, 1996). Such widespread use of phosphine over the past half century has resulted in the development of resistance among particular insect strains. Selection pressure for resistance is realized when less than ideal conditions for fumigation increase the number of applications and result in sub-lethal exposures. Fumigation within well maintained, gas tight storage facilities is rarely

\footnotetext{
* Corresponding author. School of Botany and Zoology, Australian National University, Canberra 0200, ACT, Australia. Tel.: +61 262464031.

E-mail address: james.woodman@csiro.au (J.D. Woodman).
}

achieved in many areas of the world and consequently, highly resistant strains of several major pest species have been detected in many regions and are becoming increasingly widespread (e.g. Collins et al., 2002; Lorini et al., 2007).

There is obvious importance in understanding the mode of action of phosphine and in particular, physiological responses. Most insects possess a highly efficient tracheal system with small, actively controlled openings in the cuticle (spiracles) that lead to fine branching tubules (tracheae). The regulation of gas exchange through the tracheal system permits many insect species to cope with harsh and variable environmental conditions (Hoback and Stanley, 2000). Insects that infest stored commodities are unique in facing fumigation by phosphine which enters through the spiracles and into the tracheae which ramify to directly relay respiratory gases to and from respiring cells (Nation, 2002). While hypoxia may impact upon phosphine-exposed species, relatively short fumigation times within facilities that are not usually gas tight typically prevents hypoxia from becoming uniformly severe. Additionally, low water availability is largely accommodated by 
high desiccation resistance and low inherent rates of evaporative water loss associated with a waxy epicuticle and typically low rates of respiratory transpiration (Hadley, 1994; Chown, 2002).

There have been few studies into the respiratory physiology of insects exposed to phosphine. The uptake of phosphine by an insect during fumigation is highly important in understanding how phosphine works and how insects respond (Chaudhry, 1997). Reduced phosphine uptake by a process termed active exclusion has been proposed as the primary mechanism of insecticidal resistance (Price, 1984). Reichmuth (1994) presented phosphine uptake data for both resistant and susceptible strains of the lesser grain borer, Rhyzopertha dominica. Resistant strains were observed to take up radio-labelled phosphine at a lower rate compared with susceptible strains. Susceptible individuals were immobilized in less than $1 \mathrm{~h}$ at phosphine concentrations of greater than $0.5 \mathrm{mg} / \mathrm{L}$. Importantly, similar phosphine uptake caused mortality regardless of the strain. Active respiratory control was proposed based on dead individuals of both strains passively taking up phosphine at constant and comparable levels. Further studies of insect respiratory biology are required to provide a more specific understanding of the physiological mechanisms responsible for facilitating a response to phosphine and regulating gas exchange.

Here we aim to address the need for determining the underlying regulation of the rate of $\mathrm{CO}_{2}$ release $\left(V \mathrm{CO}_{2}\right)$ and total water loss during phosphine exposure by direct measurement of ventilatory output from the central nervous system (CNS). We apply a novel technique for the simultaneous measurement of ventilatory nervous activity from the intact $\mathrm{CNS}, V_{\mathrm{CO}}$ and water loss from live specimens as described in Woodman et al. (in press). This technique was designed for relatively large specimens and we use the American cockroach, Periplaneta americana, as a model species. We report for the first time how exposure to phosphine influences the normal resting gas exchange pattern at normoxia as well as how $\mathrm{VCO}_{2}$ and water loss are regulated by the central pattern generator for ventilation in the CNS.

\section{Methods}

\subsection{Study species}

The American cockroach, P. americana, employs discontinuous gas exchange (DGE) at rest (Kestler, 1985) and has been the subject of previous neuro-physiological research identifying the metathoracic ganglion in the CNS as the primary respiratory pacemaker (Farley et al., 1967; Woodman et al., in press). Preliminary investigation determined their suitability for this research based on tolerance of $800 \mathrm{ppm}$ phosphine (v/v) in air for $8 \mathrm{~h}$. Experiments were conducted using sealed desiccator jars and no mortality or obvious sub-lethal effects were observed. Adult males were used from established breeding colonies maintained at the Australian National University.

\subsection{Recording ventilatory activity from the intact CNS}

Animals were weighed using an analytical balance (sensitivity $0.1 \mathrm{mg}$; HR-200, A\&D Co., Tokyo, Japan) and cooled to $4{ }^{\circ} \mathrm{C}$ to render them immobile. As required, each specimen was transferred onto ice (positioned ventral side up, posterior facing away) for the removal of the second leg from the left side to provide lateral access to the CNS. The animal was then centrally positioned on its back within a custom-built screw-top Perspex chamber where it was held in place with flexible adhesive over the three legs on the right side (Woodman et al., in press). Paired platinum hook electrodes $0.5 \mathrm{~mm}$ in diameter were inserted from the side of the chamber on a rigid stalk. A greased rubber seal permitted electrode insertion and subsequent fine positioning by attached micro-manipulators. The electrodes were positioned to the left of the paired nerve cord and curved forceps were used to pierce through the unsclerotised, flexible exoskeleton on each side. From the right, the paired nerve cord was gently lifted onto the electrodes which were slightly bent upwards at the tip to prevent any slipping during a trial. The electrodes were positioned anteriorly abutting the metathoracic ganglion and raised very slightly to achieve good contact. The electrodes remained in place for the duration of the experiment. High vacuum grease was used to immediately secure the lid of the chamber and ensure a gas tight seal.

The electrodes were connected to an amplifier (Powerlab 4/25T; ADInstruments, Australia) which was connected to a personal computer. Data acquisition software (Chart v.5.2.2; ADInstruments, Australia) was used to record electrical output at a frequency of 10 measurements per second before being transferred into Microsoft Excel for analysis.

\subsection{Measuring gas exchange}

The simultaneous recording of $\mathrm{CO}_{2}$ output and water flux for each individual was achieved by the incorporation of the sealed chamber into a flow-through respirometry system using a calibrated Licor 7000 differential infrared gas analyzer (Li-Cor, Lincoln, USA). Bev-a-line IV input and output tubing was connected to opposing gas fittings on the side of the chamber and an Edwards E2M-1.5 high-vacuum pump used to draw gas through the system (BOC-Edwards, BOC group Inc.). As required, experiments used either $21 \%$ oxygen in nitrogen or $800 \mathrm{ppm}$ of phosphine (at $25^{\circ} \mathrm{C}$ and 945 millibar pressure) in $21 \%$ oxygen in nitrogen passing through the chamber (internal dimensions: radius $45 \mathrm{~mm}$, height $55 \mathrm{~mm}$, volume $350 \mathrm{~cm}^{3}$ ) at a rate of $250 \mathrm{~mL} \mathrm{~min}^{-1}$ (time constant of the chamber $=84 \mathrm{~s}$, see Gray and Bradley, 2006). The experimental gas mixtures were achieved by mixing high purity oxygen, nitrogen and phosphine which passed through inline moisture and $\mathrm{CO}_{2}$ removal columns (Glass moisture trap, Model 7214; Alltech, Australia). Phosphine gas was sourced from BOC Limited (Australia) at a concentration of $2810 \mathrm{ppm}(\mathrm{v} / \mathrm{v})$ in nitrogen. Given the high toxicity and flammability of phosphine, the cylinder housing and experimental set-up was specifically designed for safe accommodation of the gas. This included modification of the Licor gas analyzer to remove several non-essential copper tubes to prevent any reaction with phosphine from the gas flow. The ratio of each gas used was controlled by a Brooks 5878 mass-flow controller with four Brooks 5850TR mass-flow meters whose calibration was checked before use using a calibrated digitial flow meter (one each for vacuum, $\mathrm{O}_{2}, \mathrm{~N}_{2}$ and $\mathrm{PH}_{3}$ ) 
a
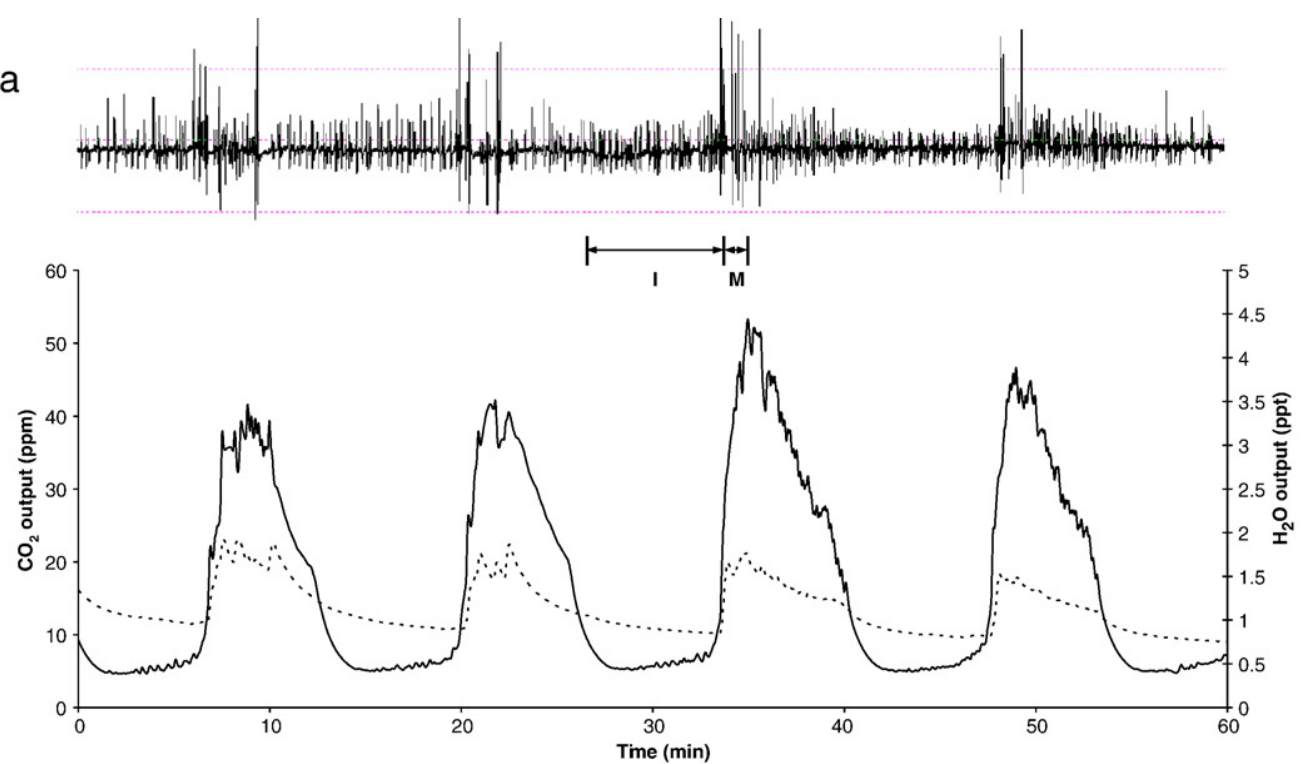

b
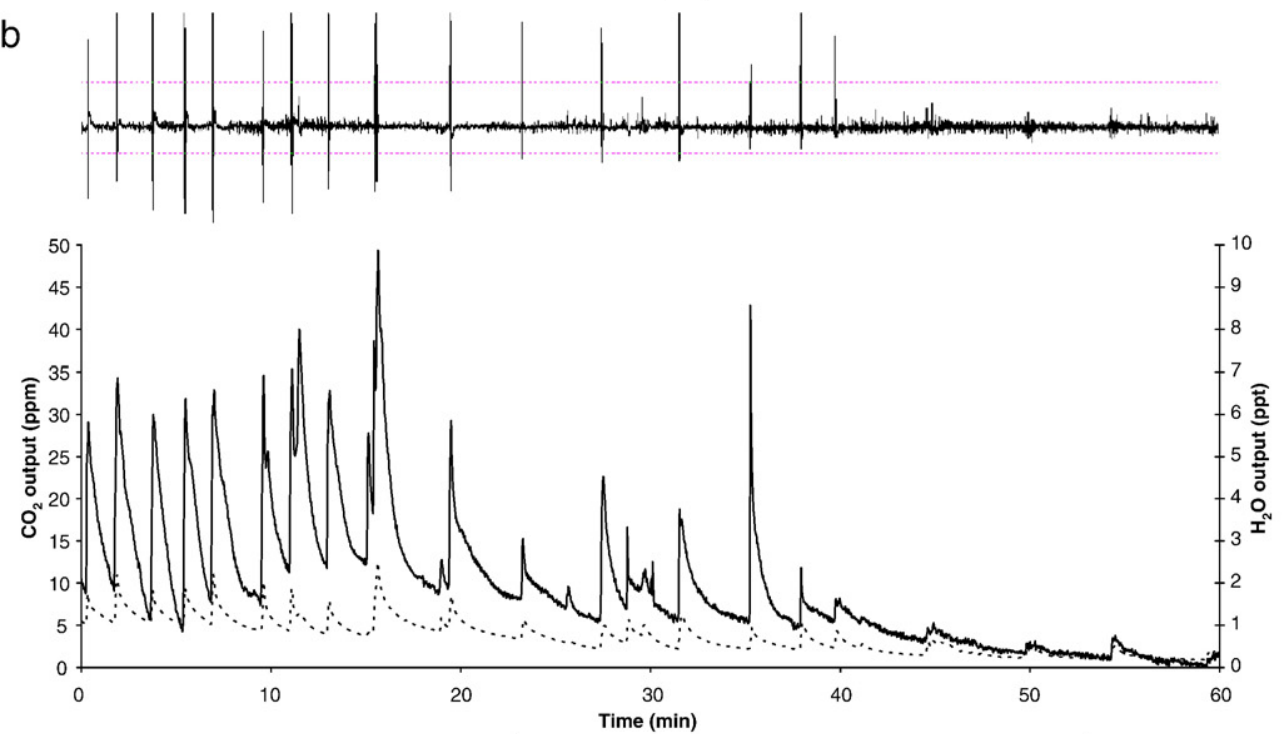

C
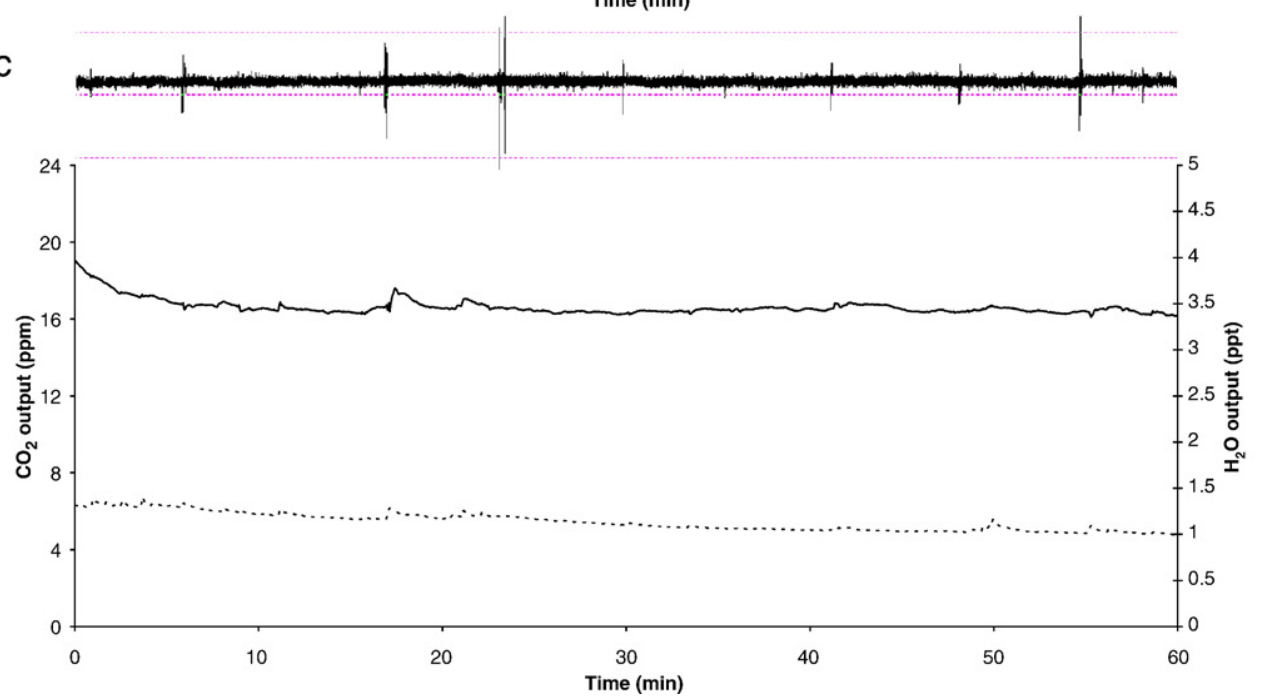

Fig. 1. Representative results showing ventilatory nervous activity from the CNS (upper trace) and simultaneous measurement of $\mathrm{CO}_{2}$ output and water loss (lower trace; solid and dotted lines respectively) over $60 \mathrm{~min}$ for P. americana at (a) normoxia before phosphine exposure, (b) the first hour of 800 ppm phosphine and (c) during the normoxic recovery period. Example major (M) and intermediate (I) CNS output spikes are shown in trace (a). $\mathrm{CO}_{2}$ release during the second hour of phosphine exposure typically resembled that of the final 20 min presented in trace (b). The occasional CNS spikes during the recovery period in trace (c) aligned with transient bodily movement. 
at $0-500 \mathrm{~mL} \min ^{-1}$ (Brooks Instruments, Hatfield, USA). The use of mass-flow controllers permitted the gas composition to be instantaneously varied as required without disruption to the gas line.

Raw data from the gas analyzer were logged on a second personal computer using Licor LI7000 data acquisition software (Version 1.0.1). Any appreciable lag time between the nervous and $\mathrm{CO}_{2}$ recordings was avoided by initiating each program simultaneously. The data from each trial were imported into Microsoft Excel for analysis.

\subsection{Treatments}

The standard pattern of gas exchange and ventilatory nervous output in $P$. americana at $21 \%$ oxygen in nitrogen is discontinuous gas exchange (DGE) (Fig. 1a) (Woodman et al., in press). For measuring the effect of phosphine gas we used a concentration of $800 \mathrm{ppm}(\mathrm{v} / \mathrm{v})$ based on preliminary data showing the animals visibly respond with transient bodily movement at this concentration, but do not experience any mortality or obvious sub-lethal effects. Experiments were conducted under dim light at $25 \pm 1{ }^{\circ} \mathrm{C}$ with 13 individual adult males. Each cockroach was exposed to $1-2 \mathrm{~h}$ of $21 \%$ oxygen in nitrogen, followed by $2 \mathrm{~h}$ of $800 \mathrm{ppm}$ phosphine and then a further $1-2 \mathrm{~h}$ of recovery at $21 \%$ oxygen. A normal resting gas exchange pattern was established before phosphine exposure.

\subsection{Data analysis and definitions}

Periods of discontinuous gas exchange whilst each animal was at rest were selected for analysis from the $\mathrm{CO}_{2}$ output data ( $n=2-6$ cycles per animal) during the initial $1-2$ hour period at normoxia. The first $30 \mathrm{~min}$ of recording was discarded due to initial water evaporation from the cuticular surface and elevated $\mathrm{CO}_{2}$ output while the animal settled. Periods of animal activity were identified from direct observation as well as perturbation in $V \mathrm{CO}_{2}$ and excluded from analysis. A DGE cycle had to exhibit an inter-burst period whereby low $\mathrm{CO}_{2}$ output was maintained, followed by a clearly defined $\mathrm{CO}_{2}$ burst phase (Fig. 1a). During phosphine exposure, the initial CNS ventilatory reaction to the gas is presented as well as $V \mathrm{CO}_{2}$, water loss and CNS output data after the initial reaction period during the second hour of exposure. Fig. 1a shows how nervous output was classified relative to $\mathrm{CO}_{2}$ release. Major CNS output was defined by the highest amplitude output spikes associated with a single $\mathrm{CO}_{2}$ burst. Intermediate CNS output spikes were defined by CNS output that was clearly differentiated from the baseline and corresponded with simultaneous $\mathrm{CO}_{2}$ release. CNS spikes (identified by either observation or the lack of a simultaneous $\mathrm{CO}_{2}$ output response) considered to correspond with nonventilatory bodily movements were excluded from analysis.

Rates of $\mathrm{CO}_{2}$ release and water loss are presented for the initial normoxic period during DGE and for the normoxic recovery period after phosphine exposure (Table 1). A zero baseline was established for all experiments at normoxia before an animal was introduced into the chamber, however, $\mathrm{CO}_{2}$ measurements were immediately and consistently decreased by
10-15 ppm upon adding phosphine into the gas flow. The baseline shift for $\mathrm{CO}_{2}$ output remained constant during phosphine use. Upon switching back to normoxia for the recovery period, the baseline readjusted to the level seen before phosphine and $\mathrm{CO}_{2}$ measurements returned to zero after the animal was removed. The integrity of the respirometry system used is established from previous and on-going work and additional closed system experiments rejected any process by which the animal may remove phosphine from the atmosphere (data not shown). The latter was tested due to the measured infrared absorption spectra of $\mathrm{CO}_{2}$ slightly overlapping with that for phosphine and suggesting that phosphine would be expected, if any effect was recorded, to elevate the $\mathrm{CO}_{2}$ baseline relative to concentration. A pressure change when switching to phosphine could have been a cause for the decreased baseline. Examination of output data from the individual measurement channels in the gas analyzer revealed an immediate shift in $\mathrm{CO}_{2}$ detection for cell A and a lesser shift for cell B after the gas had passed through the chamber. The gas analyzer is highly sensitive to changes in pressure, temperature and $\mathrm{CO}_{2}$ concentration and thus, we suggest that the different partial pressures of nitrogen and phosphine could cause the baseline shift as a result of a pressure change when phosphine is added and nitrogen reduced in the air flow. Whilst quantitative measurement of $\mathrm{CO}_{2}$ release could have been calculated during phosphine exposure, we present qualitative treatment of this data due to variation in the exact phosphine baseline among animals. Importantly, the downward baseline shift was consistent among all experiments and $\mathrm{CO}_{2}$ measurement sensitivity was not affected at any time.

\section{Results}

\subsection{Visible effects of phosphine exposure}

Exposure to $800 \mathrm{ppm}$ phosphine for $2 \mathrm{~h}$ during simultaneous measurement of CNS output and gas exchange did not cause any mortality or obvious sub-lethal effects. Animals were alive in excess of 3 days after each trial and appeared to behave normally. Initially, phosphine exposure caused transient bodily and ventilatory movements which for some individuals extended to $1 \mathrm{~h}$, followed by only occasional isolated movements. Returning the gas flow to normoxia after phosphine did not trigger any visible muscular ventilation response.

\subsection{Patterns of ventilation}

The typical gas exchange pattern of adult $P$. americana at normoxia when immobile is DGE (Fig. 1a). CNS ventilatory

Table 1

Rates of total $\mathrm{CO}_{2}$ release and evaporative water loss (mean $\pm \mathrm{SD}$ ) during normoxia before and after $800 \mathrm{ppm}$ phosphine exposure for $2 \mathrm{~h}$ at $25{ }^{\circ} \mathrm{C}$ with simultaneous CNS measurement $(n=13$ individual animals)

\begin{tabular}{lcc}
\hline & Before phosphine & After phosphine \\
\hline Mean initial mass $(\mathrm{mg})$ & $959 \pm 347$ & \\
Total $\mathrm{CO}_{2}$ output $\left(\mathrm{mL} \mathrm{h}^{-1}\right)$ & $0.23 \pm 0.08$ & $0.19 \pm 0.06$ \\
Total water loss $\left(\mathrm{mg} \mathrm{h}^{-1}\right)$ & $22.36 \pm 4.76$ & $10.51 \pm 3.78$ \\
\hline
\end{tabular}




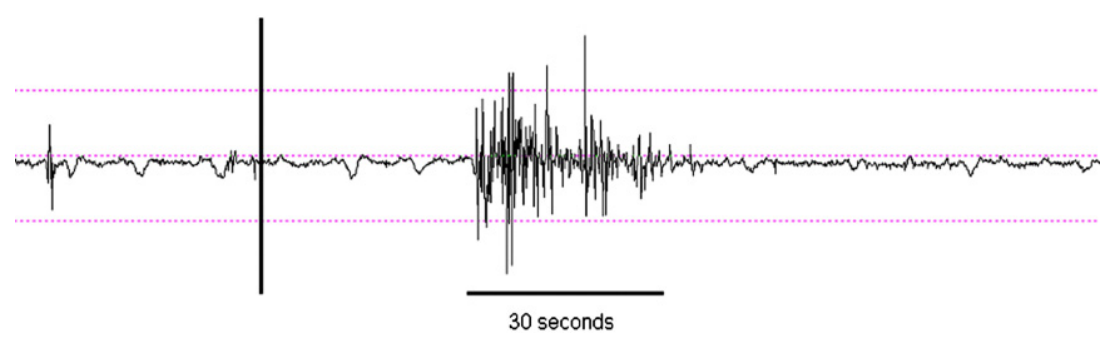

Fig. 2. A section of representative CNS recording showing the initial nervous response to 800 ppm phosphine after normoxia. The solid vertical line represents the switch to phosphine in the air flow.

output at normoxia is discretely partitioned relative to $V \mathrm{CO}_{2}$ and the phases of DGE for this species (Woodman et al., in press). Upon switching to $800 \mathrm{ppm}$ phosphine, all animals showed a CNS response within $60 \mathrm{~s}$ of introducing the gas into the system (Fig. 2). This value is conservative based on the slight lag between introducing the phosphine gas into the system and it reaching the animal. A representative CNS burst duration at first exposure to phosphine is presented in Fig. 2 and corresponded with a simultaneous burst-like release of $\mathrm{CO}_{2}$.

Phosphine exposure caused an immediate loss of DGE with short and sharp, high frequency burst-like $\mathrm{CO}_{2}$ release which varied in duration from 25 to $60 \mathrm{~min}$ (Fig. 1b). This initial hyperventilation response to phosphine was observed to decrease over a subsequent 5-10 minute period followed by lower, more stable and continuous $\mathrm{CO}_{2}$ output (Fig. 1b). Continuous gas exchange during this period was occasionally subject to $\mathrm{CO}_{2}$ release peaks either as a result of a transient ventilation event or bodily movement. After a return to normoxia following phosphine exposure (Fig. 1c), a normal discontinuous gas exchange pattern was not reinstated in any of our trials. The maximum recovery period measured was $2 \mathrm{~h}$, as a maximum period of $5 \mathrm{~h}$ per trial in total could be sustained by the nerve preparation before drying around the electrode diminished CNS recording sensitivity (Woodman et al., in press).

\subsection{Rates of carbon dioxide release and water loss}

Total $\mathrm{CO}_{2}$ release was slightly, but not significantly, reduced after phosphine exposure during the normoxic recovery period relative to periods of DGE prior to phosphine (paired sample $\mathrm{t}$-test: $\mathrm{t}=0.92, p=0.19$ ) (Table 1 ). Water loss was significantly decreased during the recovery period compared to the initial period of normoxia $(\mathrm{t}=2.11, p=0.03)$ (Table 1$)$. As a consequence of the experimental method, water loss rates were inflated, particularly during the initial period of normoxia, owing to evaporation from the dissection site which decreased as an experiment proceeded. Despite this, water loss would still be expected to be lower during the recovery period due to minimal active ventilation with greatly reduced $\mathrm{CO}_{2}$ burst frequency and intensity (Fig. 1).

\subsection{Ventilatory CNS output}

CNS output dramatically decreased after the initial reaction to phosphine and remained low during the normoxic recovery period compared to typical resting DGE (Fig. 1). Major CNS burst periods during the initial normoxic period align with the $\mathrm{CO}_{2}$ burst phase of DGE and were separated by near continuous intermediate $\mathrm{CNS}$ output during the $\mathrm{CO}_{2}$ inter-burst period (Fig. 1a). Major CNS burst periods were significantly less frequent during DGE than during phosphine exposure (Table 2). During the first hour of phosphine exposure, major CNS output spikes were more frequent, but of significantly shorter duration. Strikingly, intermediate CNS output between bursts during phosphine exposure was absent compared to that at normoxia prior to phosphine, indicating negligible active inter-burst ventilation (Fig. 1b, Table 2). During the normoxic recovery period CNS output remained low and stable, except for a small proportion of animals (4 of 13) that showed some active ventilation and simultaneous $\mathrm{CO}_{2}$ release bursts that were of lower intensity than those during DGE. For these animals, the $\mathrm{CO}_{2}$ bursts were more common during the second hour of recovery and accounted for an average of $32 \%$ of the total recovery time period.

\section{Discussion}

Influenced by metabolic, hormonal, sensory, neural and behavioural factors, gas exchange in insects is regulated by output from a central pattern generator $(\mathrm{CPG})$ for ventilation in the CNS (Bustami and Hustert, 2000; Nation, 2002). A 2 hour exposure to $800 \mathrm{ppm}$ phosphine in $P$. americana does not cause mortality or any obvious sub-lethal effects, but does immediately disrupt the normal gas exchange pattern. Importantly, this species is not adapted to phosphine exposure and we report a response to the gas in a species that is inherently tolerant to at least short term

Table 2

Ventilatory nervous activity from the CNS in P. americana $(n=13)$ during rest

\begin{tabular}{lcrr}
\hline & $\begin{array}{l}\text { Initial } \\
\text { normoxia }\end{array}$ & Phosphine & $\begin{array}{r}\text { Recovery } \\
\text { normoxia }\end{array}$ \\
\hline No. of major CNS burst periods & $4.2 \pm 1.9$ & $15.1 \pm 6.3$ & $3.0 \pm 2.2$ \\
Major CNS burst duration (s) & $85.2 \pm 20.6$ & $8.5 \pm 4.2$ & $15.7 \pm 8.9$ \\
No. of intermediate CNS bursts & $97.4 \pm 20.3$ & $11.9 \pm 5.6$ & $7.1 \pm 3.3$ \\
Intermediate CNS burst duration (s) & $11.8 \pm 6.9$ & $14.7 \pm 3.2$ & $12.0 \pm 3.5$ \\
\hline
\end{tabular}

Only CNS data corresponding with $\mathrm{CO}_{2}$ release from simultaneous measurements were used at normoxia, $800 \mathrm{ppm}$ phosphine and during normoxic recovery. Major CNS bursts during DGE at initial normoxia are grouped together for each $\mathrm{CO}_{2}$ burst period. Data are presented as the mean $\pm \mathrm{SD}$ with the number of CNS bursts calculated per hour. 
exposure. For $P$. americana, phosphine induced an almost immediate $(<60 \mathrm{~s})$ ventilatory response from the metathoracic ganglion in the CNS consistent with simultaneous sharp peaks in $\mathrm{CO}_{2}$ release and the loss of the normal respiratory pattern (DGE) (Fig. 1b). An almost immediate CNS reaction to phosphine is consistent with that previously recorded for a change in oxygen tension in this species (Woodman et al., in press), although oxygen remained constant at $21 \%$ during phosphine exposures. DGE was not observed at any time during a phosphine treatment and following the initial reaction to the gas, $V \mathrm{CO}_{2}$ was continuous with the exception of occasional movement associated output.

In most stored-product insects, exposure to high concentrations of phosphine induces a state of narcosis whereby the animal is completely immobilized (Chaudhry, 1997). For adult red flour beetles (Tribolium castaneum), narcosis may infer some protection against the insecticidal action of phosphine by lowering respiration and metabolic activity, particularly during short exposures (Winks, 1985; Chaudhry, 1997). The possibility of a poisoning effect in $P$. americana cannot be completely dismissed, however we assert an active physiological response based on the retained potential for movement at all times during exposure. A similar ventilatory response to that observed for phosphine (after the initial hyper-ventilation response) has been previously reported for $P$. americana when exposed to severe hypoxia ( $2 \%$ oxygen) (Woodman et al., in press). It is possible that the ready reaction of phophine with dissolved oxygen may deplete cellular oxygen and result in the CNS sensing the effects of phosphine as those associated with hypoxia, in addition to any possible poisoning effect.

$V \mathrm{CO}_{2}$ during the 2 hour normoxic recovery period was slightly decreased compared to the initial 2 hour period at normoxia as a result of phosphine exposure (Table 1). A simultaneous reduction in water loss supports the proposal of lowered metabolic activity in response to phosphine whereby CNS output and continuous $\mathrm{CO}_{2}$ release show minimal active ventilation and only occasional abdominal pumping movements. During recovery after phosphine exposure, we did not observe a return to a normal discontinuous gas exchange pattern. Some individuals showed what could be interpreted as the beginnings of a cyclic $\mathrm{CO}_{2}$ release pattern, but the typical resting respiratory pattern with clearly defined phases was not observed. The experimental method used did not permit measurements beyond a total of $5 \mathrm{~h}$ (Woodman et al., in press), however given lively specimen condition, we measured normoxic gas exchange 3 days after exposure without CNS recording. Results showed DGE is reinstated as the default resting respiratory pattern at some point beyond the 2 hour recovery period (data not shown).

The active exclusion hypothesis for reduced phosphine uptake in resistant compared to susceptible insect populations has been tested using radioactive fumigants to trace uptake (Price, 1981; Price \& Dance, 1983; Nakakita \& Kuroda, 1986; Chaudhry \& Price, 1990) as well as by measuring differences in metabolic activity (Nakakita et al., 1974; Price, 1980; Winks, 1984; Chaudhry et al., 2004; Pimentel et al., 2007). Price (1980) reported a marked reduction in oxygen consumption by a susceptible strain of Rhyzopertha dominica at 200 ppm phosphine that was further reduced during exposure to 400 and $800 \mathrm{ppm}$ respectively. At $800 \mathrm{ppm}$, oxygen consumption dropped to near zero with all individuals rendered immobile. Recovery to a normal respiratory state only occurred after prolonged airing, but recovery was often incomplete with the animals remaining in a state of narcosis. It is therefore interesting that although $P$. americana is not adapted to phosphine exposure, it is tolerant to $800 \mathrm{ppm}$ without losing the capacity for movement. Data for $P$. americana contrast with that for susceptible $R$. dominica whereby at $800 \mathrm{ppm}$ phosphine, $P$. americana remained alert and showed only a slight reduction in $\mathrm{CO}_{2}$ release compared with the almost total cessation of metabolic activity in $R$. dominica (Price, 1980).

The initial reaction to phosphine showed high frequency major CNS bursts aligning with $\mathrm{CO}_{2}$ output peaks, but no intermediate CNS output. After this initial hyper-ventilation response, it appears likely that $\mathrm{CO}_{2}$ release in $P$. americana is similar to that during the normoxic recovery period and lower than that during DGE at normoxia (Fig. 1). The baseline shift during phosphine exposure precluded output rate calculations, however low and stable evaporative water loss as well as negligible intermediate ventilatory CNS output supports minimal active ventilation during phosphine compared to normal resting gas exchange at normoxia (Fig. 1a,b). Reduced $\mathrm{CO}_{2}$ release in cockroaches whilst exposed to phosphine indicates a lower metabolic rate, assuming that decreased $V \mathrm{CO}_{2}$ is indicative of reduced oxygen consumption. For $P$. americana, tolerance to phosphine appears to be facilitated by the CNS sensing phosphine and remaining able to exploit the inherent flexibility of the tracheal system without active ventilatory movements. To further clarify how $\mathrm{CO}_{2}$ output is affected during phosphine exposure, research is needed to quantify the rate of $\mathrm{CO}_{2}$ release during a phosphine treatment. For example, this could be achieved using closed system respirometry to avoid the baseline shift recorded here using a high sensitivity flow-through system.

The use of phosphine as the predominant fumigant for treating stored commodities against insect infestation will continue into the foreseeable future. The decreasing efficacy of phosphine due to resistance development has placed increased importance on understanding the physiological response in insects. Here we have shown how the ventilatory pacemaker in the CNS responds to phosphine and how gas exchange is subsequently modulated in a model insect species. The active exclusion of phosphine by reduced gas exchange warrants further quantitative research in stored product insects, as does discrimination between muscular ventilation and spiracular control during exposure. Additionally, the biochemical mode of action of the fumigant must be further explored to achieve an integrative and comprehensive understanding.

\section{Acknowledgements}

For technical and engineering assistance we thank Greg Dojchinov and Stuart Alley (CSIRO Entomology). This research was financially supported by a $\mathrm{PhD}$ scholarship awarded to J.D.W. by the Bulk Handling Companies of Australia.

All experiments as part of the research presented herein were conducted in compliance with the current laws of Australia. 


\section{References}

Bustami, H.P., Hustert, R., 2000. Typical ventilatory pattern of the intact locust is produced by the isolated CNS. J. Insect Physiol. 46, 1285-1293.

Chaudhry, M.Q., 1997. A review of the mechanisms involved in the action of phosphine as an insecticide and phosphine resistance in stored-product insects. Pestic. Sci. 49, 213-228.

Chaudhry, M.Q., Bell, H.A., Savvidou, N., MacNicoll, A.D., 2004. Effect of low temperatures on the rate of respiration and uptake of phosphine in different life stages of the cigarette beetle Lasioderma serricorne (F.). J. Stored. Prod. Res. 40, 125-134.

Chaudhry, M.Q., Price, N.R., 1990. Insect mortality at doses of phosphine which produce equal uptake in susceptible and resistant strains of Rhyzopertha dominica (F.) (Coleoptera: Bostrichidae). J. Stored. Prod. Res. 26, 101-107.

Chown, S.L., 2002. Respiratory water loss in insects. Comp. Biochem. Physiol. A $133,791-804$.

Farley, R.D., Case, J.F., Roeder, K.D., 1967. Pacemaker for tracheal ventilation in the cockroach, Periplaneta americana (L.). J. Insect. Physiol. 13, 1713-1728.

Collins, P.J., Daglish, G.J., Bengston, M., Lambkin, T.M., Pavic, H., 2002. Genetics of resistance to phosphine in Rhyzopertha dominica (Coleoptera: Bostrichidae). J. Econ. Entomol. 95, 862-869.

Gray, E.M., Bradley, T.J., 2006. Evidence from mosquitoes suggests that cyclic gas exchange and discontinuous gas exchange are two manifestations of a single respiratory pattern. J. Exp. Biol. 209, 1603-1611.

Hadley, N.F., 1994. Water relations of terrestrial arthropods. Academic press Inc., San Diego, California.

Hoback, W.W., Stanley, D.W., 2000. Insects in hypoxia. J. Insect. Physiol. 47, 533-542.

Kestler, P., 1985. Respiration and respiratory water loss. Environmental physiology and biochemistry of insects. Springer, Berlin.

Lorini, I., Collins, P.J., Daglish, D.J., Nayak, M.K., Pavic, H., 2007. Detection and characterisation of strong resistance to phosphine in Brazilian Rhyzopertha dominica (F.) (Coleoptera: Bostrychidae). Pest. Manag. Sci. 63, 358-364.
Nakakita, H., Kuroda, J., 1986. Differences in phosphine uptake between susceptible and resistant strains of insects. J. Pestic. Sci. 11, 21-26.

Nakakita, H., Saito, T., Iyatomi, K., 1974. Effect of phosphine on the respiration of adult Sitophilus zeamais. J. Stored. Prod. Res. 10, 87-92.

Nation, J.L., 2002. Insect physiology and biochemistry. CRC Press, Florida.

Pimentel, M.A.G., Faroni, L.R.D.'A., Totola, M.R., Guedes, R.N.C., 2007. Phosphine resistance, respiration rate and fitness consequences in stroedproduct insects. Pest. Manag. Sci. 63, 876-881.

Price, N.R., 1980. The effect of phosphine on respiration and mitochondrial oxidation in susceptible and resistant strains of Rhyzopertha dominica. Insect Biochem. 10, 65-71.

Price, N.R., 1981. A comparison of the uptake and metabolism of resistance in Phyzopertha dominica (F.) (Coleoptera: Bostrichidae). Comp. Biochem. Physiol. C 69, 129-131.

Price, N.R., 1984. Active exclusion of phosphine as a mechanism of resistance in Rhyzopertha dominica (F.) (Coleoptera: Bostrichidae). J. Stored. Prod. Res. 20, 163-168.

Price, N.R., Dance, S.J., 1983. Some biochemical aspects of phosphine action and resistance in three species of stored product beetles. Comp. Biochem. Physiol. C 76, 277-281.

Reichmuth, C., 1994. Uptake of phosphine by stored-product pest insects during fumigation. Proceedings of the 6th International Working Conference on Stored-product Protection vol. 1, 157-162.

Winks, R.G., 1984. The toxicity of phosphine to adults of Tribolium Castaneum (Herbst): time as a dosage factor. J. Stored. Prod. Res. 20, 45-56.

Winks, R.G., 1985. The toxicity of phosphine to adults of Tribolium Castaneum (Herbst): phosphine induced narcosis. J. Stored. Prod. Res. 21, 25-31.

Woodman, J.D., Cooper, P.D., Haritos, V.S., in press. The nervous regulation of discontinuous gas exchange in intact Periplaneta americana. Comp. Biochem. Physiol. C. 